Yully Graciela Bayona Madrid ${ }^{1}$ Abraham Meneses López ${ }^{2}$

${ }^{1}$ Cirujano Dentista

Docente del Departamento Académico de

Estomatología del Niño y del Adolescente. Facultad de Estomatología. Universidad Peruana Cayetano Heredia.

\section{Correspondencia}

Yully Graciela Bayona Madrid Jr. Joaquín Turina 250-302 Urb. Las Magnolias Lima 41 , Perú

Teléfono: 2245272/981119537

e-mail: poohpiura@ hotmail.com

Recibido :26 de setiembre del 2008

Aceptado : 15 de abril del 2009

\title{
Procedimientos clínicos para la corrección de la discrepancia de masa dentaria en pacientes con maloclusión clase I
}

Bayona-Madrid YG, Meneses-López A. Procedimientos clínicos para la corrección de la discrepancia de masa dentaria en pacientes con maloclusión clase I. Rev Estomatol Herediana. $2010 ; 20(1): 13-18$.

\section{RESUMEN}

El objetivo de este estudio fue determinar la discrepancia de masa dentaria, su ubicación, los procedimientos clínicos utilizados para su corrección y las relaciones oclusales finales obtenidas en pacientes con maloclusión clase I tratados ortodónticamente en el servicio de Postgrado de la Universidad Peruana Cayetano Heredia (UPCH). Se evaluaron 30 historias clínicas con modelos iniciales y finales de pacientes con maloclusión clase I que recibieron tratamiento sin extracciones el servicio de ortodoncia de la clínica dental de la UPCH. Se observó que el 60\% del total de pacientes presentaron al inicio una discrepancia de Bolton total no significativa y un 53\% presentaron discrepancia anterior. La ubicación de la discrepancia inicial se encontró en la arcada superior. También se encontró que el procedimiento de corrección más utilizado (57\%) fue el desgaste proximal. Todos los casos, independientemente de la discrepancia de masa dentaria, terminaron con relaciones oclusales aceptables. Conclusiones: La presencia de discrepancia de masa dentaria anterior fue mayor a la discrepancia total. La discrepancia total se ubicó con mayor frecuencia en la arcada superior y la discrepancia anterior en la arcada inferior. El tratamiento mas frecuente fue el desgaste proximal y las relaciones oclusales fueron adecuadas tanto en pacientes con o sin discrepancia de masa dentaria.

Palabras clave: MALOCLUSIÓN DE ANGLE CLASE I / ORTODONCIA.

Clinical procederes for the correction of tooth mass discrepancy in Class I malocclusion patients

ABSTRACT

The purpose of this study was to determine the discrepancy of tooth size, its location, the clinical procedures used for its correction and the final occlusal relationships in patients with Class I malocclusion treated orthodontically in the Post graduate service at the Cayetano Heredia Peruvian University (UPCH).Thirty dental charts with initial and final dental casts were evaluated in patients with class I malocclusion who were treated without extractions at the department of Orthodontics of the dental clinic of UPCH. It was observed that $60 \%$ of the patients had at the beginning a total non-significant discrepancy of Bolton and $53 \%$ of them had an anterior discrepancy. The location of the initial discrepancy was located in the superior arch. It was also found that the most common procedure of correction (57\%) was stripping . All the cases, regardless of the tooth size discrepancy, ended in acceptable occlusal relationships. Conclusions: The presence of the tooth size anterior discrepancy was higher than the total discrepancy. The total discrepancy was frequently located in the superior arch and the anterior discrepancy in the inferior arch. The most common treatment was stripping and the occlusal relationships were adequate for patients with and without tooth size discrepancy.

Keywords: ANGLE CLASS I MALOCCLUSION / ORTHODONTICS.

\section{Introducción}

El objetivo de un plan de tratamiento en ortodoncia es garantizar la función oclusal y la estética que puede ser afectado por diferentes factores que originan una maloclusión, entre ellos, la discrepancia de masa dentaria (1).

Uno de los primeros investigadores que realizó mediciones del tamaño dentario en humanos fue Black, en el siglo XIX. Las tablas de promedio de dichas mediciones son usadas aún como referencia $(2,3)$. Los estudios de Black (1902) y Neff $(1949)(2,4,5)$ fueron seguidos por Bolton, quién halló la relación de masa dentaria entre dientes inferiores y superiores en modelos con oclusión óptima; y comparó la suma de sus anchos mesiodistales. Determinó una proporción total de 91,3 (de $+1,91$ ) y anterior de canino a canino en 77,2 (de $+1,65$ ).

Bernabé et al. (6) determinaron la proporción dentaria de los dientes superiores e inferiores en 200 niños con dentición permanente completa, sin lesiones cariosas o restauraciones interproximales. Concluyeron que la discrepancia anterior entre hombres y mujeres no presentó diferencia estadísticamente significativa mientras que en la discrepancia total sí fue significativa.

Para elaborar un plan de tratamiento se tiene en consideración la proporción de la discrepancia de masa dentaria y procedimientos de compensación estética como restauraciones, reconstrucción protésica, desgaste proximal y coronas cerámicas (7-10). Los problemas de exceso de masa dentaria antero inferior han sido resueltos por diversos procedimientos, es decir, el paciente podría ser tratado para reducir el overbite y el overjet incrementando el torque lingual de las piezas anteriores superiores teniendo en cuenta 
siempre los límites funcionales (movimientos excursivos) y estéticos. Otra alternativa de tratamiento es dejar espacios a distal de los incisivos laterales superiores, este espacio puede ser reducido con una inclinación de la raíz hacia distal con la finalidad de aumentar el ancho de los incisivos, pero no debemos olvidar que existen límites estéticos. Una tercera alternativa es disminuir el ancho de los incisivos inferiores para reducir el grosor del esmalte, teniendo en cuenta la proximidad de las raíces. Generalmente el desgaste de 2 a 2,5mm de esmalte dividido entre las diez superficies (mesial de canino a canino) es el límite de esta técnica. Finalmente, las piezas antero superiores serían reconstruidas con material resinoso para aumentar su ancho mesiodistal $(11,12)$.

El desgaste interproximal se puede realizar por medios mecánicos con discos abrasivos para pieza de mano de baja velocidad, la unidad de ultrasonido haciendo uso de una pasta de hidróxido de aluminio con una punta especial y tiras de lija. Por medios químicos con ácido fosfórico al 37\%, o combinados. Joseph et al. $(11,13,14)$ reportaron que la combinación del sistema químico (ácido fosfórico 37\%) y mecánico crea en el esmalte una superficie blanda que presentaría un alto potencial para la remineralización, lo que sería beneficioso para las piezas dentarias. Entre las ventajas que representa el desgaste mesiodistal de la corona está mejorar la relación del overbite y overjet de los dientes anteriores es decir, mejorar la función mutuamente protegida (13).

Di paolo y Boruchov (15) señalan la importancia de la medición del esmalte interproximal y la longitud de la raíz en relación a la corona a través de una radiografía periapical como método de evaluación.
Señalan que el límite del desgaste de la corona debe ser la mitad del esmalte interproximal para no originar problemas periodontales.

La innovación de técnicas y materiales resinosos han hecho posible otra alternativa como una solución semipermanente al exceso de masa dentaria anteroinferior. La técnica de ácido grabador para restauraciones y la reconstrucción estética con resina sin preparación mecánica proporciona un excelente sellado con mínima filtración marginal. La resina proporciona retención, un límite lingual, incisal, proximal, y un aceptable acabado vestibular así como una textura aceptable. Por lo tanto esta técnica de incremento con material resinoso para corregir la discrepancia de masa dentaria debe ser planificada dentro del tratamiento, para obtener estética y función (16).

Heusdens et al. (17) realizaron un estudio experimental para comparar la discrepancia de masa dentaria total y anterior según los valores de Bolton con los reportados por otros estudios epidemiológicos y evaluar la exactitud de las mediciones de la discrepancia de masa dentaria y entre otros objetivos, evaluar el efecto de la terapia de extracciones de cuatro premolares en la oclusión. Los resultados obtenidos concluyen que no existe diferencia significativa entre la discrepancia de masa dentaria total de los valores de Bolton comparados con los cuatro estudios sin embargo la proporción anterior significativa mente diferente, debido a la gran variabilidad morfológica del ancho de los incisivos superiores, alta reproducibilidad (99\%) de las mediciones de la discrepancia de masa dentaria y la discrepancia de masa severa afecta ligeramente a la oclusión porque en los casos que presentaron una discrepancia severa aún así presentaron relaciones molares de clase I, overjet y overbite aceptables. Además concluyeron que una curva de Spee de profundidad de $6 \mathrm{~mm}$ genera pobres resultados y la terapia de extracciones afecta ligeramente en la oclusión final.

Es necesario tener en cuenta que si se presenta alteración de la proporción de masa dentaria no va a influir en el overjet, overbite, la inclinación, el ángulo interincisal. Asimismo, el grosor del diente va a ejercer influencia en la relación de masa dentaria ideal (18).

El propósito de este estudio fue determinar la discrepancia de masa dentaria y su ubicación y, los procedimientos clínicos para su corrección así como, evaluar sus relaciones oclusales finales, en pacientes con maloclusión clase I tratados ortodónticamente en el servicio de Postgrado de la Universidad Peruana Cayetano Heredia.

\section{Material y métodos}

Se seleccionaron pacientes con maloclusión de clase I del servicio de Ortodoncia de la Universidad Peruana Cayetano Heredia. Treinta casos en dentición permanente con historia clínica completa y con tratamiento ortodóntico sin extracciones y sus respectivos modelos inicial y final, sin presentar caries dental interproximal visible, restauraciones interproximales, o anomalías dentales.

El examinador fue calibrado en modelos elegidos al azar con la finalidad de comparar las mediciones realizadas por el examinador y su asesor. Se midieron dos veces cada diente, desde la primera molar derecha hasta la primera molar izquierda. Si la 
diferencia era menor de $0,5 \mathrm{~mm}$; la primera medición era registrada. Sin embargo, si la medición era mayor de $0,5 \mathrm{~mm}$ se realizó otra medición y esta era registrada. La medición se realizó en 10 pares de modelos por día con la finalidad de evitar la fatiga visual. Después de haber realizado las mediciones correspondientes se procedió a sumar los anchos mesiodistales de las 6 piezas anterosuperiores, antero-inferiores y las 12 piezas superiores e inferiores, y se aplicó las fórmulas de Bolton. El análisis estadístico se realizó con la prueba de asociación Chi cuadrado y distribución de frecuencias.

\section{Resultados}

En la tabla 1 se observa que del total de pacientes el 60\%(18) presentaron al inicio una discrepancia de Bolton total no significativa y el $77 \%(23)$ presentaron una discrepancia de Bolton total no significativa al final del tratamiento.

La tabla 2 muestra la distribución de los pacientes según la discrepancia de Bolton anterior. El 53\% del total de pacientes (16) presentaron al inicio del tratamiento una discrepancia de Bolton anterior significativa, sin embargo al finalizar el tratamiento el porcentaje disminuyó a un 37\%(11); viéndose incrementada la discrepancia de Bolton anterior no significativa final a $63 \%(19)$.

En la tabla 3 se señala la distribución de la discrepancia de Bolton según ubicación. Del total de pacientes el $60 \%(18)$ presentaron discrepancia de Bolton total inicial en la arcada superior; al final del tratamiento aumentó el porcentaje a $76,7 \%$ (23) de la discrepancia en la misma arcada. En lo que respecta a la discrepancia de Bolton anterior al iniciar el tratamiento, en el 60\%(18) se encontró en la arcada inferior pero al finalizar el tratamiento dicha discrepancia aumentó en la arcada superior a un 53,3\%(16).

En la tabla 4 se indica el tratamiento realizado. Se observa que del $60 \%$ de pacientes que presentaron al inicio del tratamiento una discrepancia de Bolton total no significativa, al 30\%(9 pacientes) se les realizó desgaste interproximal, el 23,3\%(7) no recibieron ningún tratamiento y el 6,7\%(2) se les realizó una combinación de desgaste interproximal con incremento de resina. Asimismo el 53\% de la muestra que presentó al inicio del tratamiento una discrepancia de Bolton anterior significativa recibió el 36,4\%(11) desgaste interproximal, el $10 \%(3)$ no recibió tratamiento y el 6,6\%(2) la combinación de desgaste interproximal con incremento de resina.

En la tabla 5 se muestra que del

Tabla 1. Discrepancia de masa dentaria total.

\begin{tabular}{lccrc}
\hline & \multicolumn{2}{c}{ Bolton total inicial } & \multicolumn{2}{c}{ Bolton total final } \\
& $\mathrm{n}$ & $(\%)$ & $\mathrm{n}$ & $(\%)$ \\
\hline No significativo & 18 & $(60)$ & 23 & $(77)$ \\
Significativo & 12 & $(40)$ & 7 & $(23)$ \\
Total & 30 & $(100)$ & 30 & $(100)$ \\
\hline
\end{tabular}

Tabla 2. Discrepancia de masa dentaria anterior.

\begin{tabular}{lcccc}
\hline & \multicolumn{2}{c}{ Bolton total inicial } & \multicolumn{2}{c}{ Bolton total final } \\
& $\mathrm{n}$ & $(\%)$ & $\mathrm{n}$ & $(\%)$ \\
\hline No significativo & 14 & $(47)$ & 19 & $(63)$ \\
Significativo & 16 & $(53)$ & 11 & $(37)$ \\
Total & 30 & $(100)$ & $30(100)$ \\
\hline
\end{tabular}

Tabla 3. Ubicación de la discrepancia de masa dentaria.

\begin{tabular}{|c|c|c|c|c|c|c|}
\hline & \multirow{2}{*}{\multicolumn{2}{|c|}{$\begin{array}{l}\text { Bolton total } \\
\text { inicial }\end{array}$}} & & \multicolumn{3}{|c|}{ Bolton anterior } \\
\hline & & & final & inicial & final & \\
\hline & $\mathrm{n}$ & $(\%)$ & n $\quad(\%)$ & $\mathrm{n} \quad(\%)$ & $\mathrm{n}$ & (\%) \\
\hline Superior & 18 & (60) & $23(76,7)$ & (40) & 16 & (14) \\
\hline Inferior & 12 & (40) & $7(23,3)$ & $18 \quad(60)$ & 14 & (46) \\
\hline Total & 30 & (100) & $30(100)$ & $30(100)$ & 30 & (100) \\
\hline
\end{tabular}

Tabla 4. Tratamiento realizado en la fase de acabado según discrepancia de masa dentaria.

\begin{tabular}{|c|c|c|c|c|c|}
\hline & \multicolumn{2}{|c|}{ Bolton total inicial } & \multicolumn{3}{|c|}{ Bolton anterior inicial } \\
\hline & NS & $\mathrm{S}$ & Ns & & $\mathrm{S}$ \\
\hline & n $\quad(\%)$ & $(\%)$ & $\mathrm{n}$ & $(\%)$ & (\%) \\
\hline Desgaste interproximal & $9(30,0)$ & $8(26,7)$ & 6 & $(20,1)$ & $11(36,4)$ \\
\hline Sin tratamiento & $7(23,3)$ & $3(10,0)$ & 7 & $(23,5)$ & $3(10,0)$ \\
\hline Desgaste interproximal y resina & а $2(6,7)$ & $1(3,3)$ & 1 & $(3,4)$ & $2(6,6)$ \\
\hline Total & $18(60,0)$ & $12(40,0)$ & 14 & $(47,0)$ & $16(53,0)$ \\
\hline
\end{tabular}

NS: no significativo; S: significativo

Tabla 5. Relaciones oclusales según discrepancia de masa dentaria inicial total.

\begin{tabular}{llll}
\hline Relaciones & $\begin{array}{l}\text { No significativo } \\
\text { oclusales finales }\end{array}$ & $\begin{array}{l}\text { Significativo } \\
\mathrm{n}(\%)(\% \text { BTI) }\end{array}$ & $\begin{array}{l}\text { Total } \\
\mathrm{n}(\%)(\% \mathrm{BTI})\end{array}$ \\
$\mathrm{n}(\%)(\% \mathrm{BTI})$ \\
\hline Relaciones adecuadas & $10(52,6)(55,6)$ & $9(47,4)(75)$ & $19(63,3)$ \\
Relaciones inadecuadas & $8(72,7)$ & $3(27,3)(25)$ & $11(36,7)$ \\
Total & $18(60)$ & $12(40)$ & $30(100)(100)$ \\
\hline
\end{tabular}

BTI: Bolton Total Inicial 
total de pacientes que terminaron con buenas relaciones oclusales (19), el $52,6 \%$ presentaron al inicio una discrepancia total no significativa, sin embargo, el 55,6\% de los pacientes que presentaron una discrepancia de Bolton total no significativa terminaron con buenas relaciones oclusales. Así mismo, del total de pacientes (11) que terminaron el tratamiento con inadecuadas relaciones oclusales, el $72,7 \%$ presentaron al inicio una discrepancia de Bolton total no significativa, ellos representaban el $44,4 \%$ del total de casos con discrepancia de Bolton total no significativa. De los casos con discrepancia de Bolton total significativa, el 75\%(9) terminaron con buenas relaciones oclusales.

\section{Discusión}

La muestra constó de 30 pacientes con maloclusión clase I que recibieron tratamiento sin extracciones atendidos en la Clínica Dental de la Universidad Cayetano Heredia durante el periodo $2003 \mathrm{al}$ 2008, de los cuales 18 pacientes (60\%) presentaron discrepancia de Bolton total inicial no significativa y 12 pacientes (40\%) discrepancia de Bolton significativa. Así mismo, 14 pacientes $(47 \%)$ presentaron discrepancia de Bolton anterior inicial no significativa y 16 pacientes (53\%) una discrepancia de Bolton significativa. Se encontró que existía una correlación entre la discrepancia de Bolton total inicial y final $(r=0,515)$ siendo a su vez esta relación estadísticamente significativa $(\mathrm{p}=0,004)$.

Bolton (2) demostró que la correlación entre la discrepancia anterior y la total, fue de 0,5 ; no significativa; sin embargo Él concluyó que fue suficientemente significativo para introducir el análisis. Por el contrario White reportó una baja correlación $(r=0,12)$ entre la discrepancia de Bolton total y anterior. Al-Tamini (19) halló una correlación $(r=0,59)$ similar a Bolton. En el presente estudio también se encontró una baja correlación entre la discrepancia de Bolton total y anterior iniciales ( $r=-0,055)$; y la discrepancia Bolton total y anterior finales $(r=0,234)$. Esta correlación puede deberse a la distribución de la muestra según la discrepancia de Bolton inicial y final es decir, si distribuimos la muestra según la discrepancia de Bolton total inicial vamos a obtener una muestra con un $60 \%$ de pacientes que presentan una discrepancia total no significativa; y sin embargo, si distribuimos la muestra según la discrepancia de Bolton anterior inicial encontramos una muestra con un $53 \%$ de pacientes que presentan discrepancia de Bolton anterior significativa lo mismo ocurre con las discrepancias de Bolton finales ; lo que explica la baja correlación entre estas variables.

El presente estudio encuentra que la arcada superior es la ubicación más común de la discrepancia de Bolton total inicial en el $60 \%$ de la muestra. Así mismo se comprobó que existe una correlación entre la ubicación de la discrepancia de Bolton total inicial y la ubicación de la discrepancia de Bolton total final $(r=0,515)$ presentando a su vez una relación estadísticamente significativa $(p=0,004)$. Igualmente, se estableció una correlación entra la ubicación de la discrepancia de Bolton total inicial y la ubicación de la discrepancia de Bolton anterior inicial $(r=0,528)$ con una relación estadísticamente significativa $(\mathrm{p}=0,003)$.

En el estudio realizado por Freeman et al. (20) se determinó que la discrepancia de Bolton total fue casi igual en la arcada superior como en la arcada inferior, mientras que la discrepancia de masa dentaria anterior fue casi dos veces más $(19,7 \%)$ en la arcada inferior que en la arcada superior (10,8\%). Santero (4) y Araujo, Souki (21) encontraron similares resultados a los de Freeman.

En la práctica clínica muchas veces la corrección de la discrepancia de masa dentaria se corrige realizando desgaste proximal o incrementando con material resinoso a las piezas dentarias (3,15,17-19,22,23).

El stripping, conocido como la acción de remover el esmalte interproximal, tiene como propósito crear espacio para realizar el tratamiento ortodóntico, dar forma y tamaño adecuado a las piezas dentarias. También se le conoce con los siguientes nombres: salandering, slicing, Hollywood trim, desgaste selectivo, reducción mesiodistal, reaproximación, interproximal wear y coronoplastía (24).

Moreno (24) señala que entre las principales indicaciones para realizar desgaste proximal se encuentra la discrepancia de masa dentaria. Ballard en 1944 recomendó tener cuidado al realizar stripping en las superficies interproximales de los dientes anteriores cuando existe un desbalance o desequilibrio. Sheridan (18) indica que es posible alcanzar excelentes relaciones inter-incisales y oclusales realizando stripping.

La innovación de materiales y la aparición de nuevas técnicas han hecho posible otra alternativa como una solución semipermanente para corregir la discrepancia de masa. La técnica de ácido grabador para restauraciones y la reconstrucción estética con resina sin preparación mecánica proporciona un excelente 
sellado con mínima filtración marginal.

En el presente estudio se encuentra que, predominantemente, se realizaron dos tratamientos durante la fase de acabado, stripping e incremento de resina, de los pacientes que se les realizó stripping la mayoría presentaron discrepancia anterior inicial significativa; lo mismo ocurrió en aquellos pacientes que recibieron desgastes proximales con incremento de resina.

Los pacientes que presentaron adecuadas relaciones oclusales al final del tratamiento, que fue la mayor parte de la muestra, presentaron discrepancia de Bolton total inicial no significativa, esto fue similar en los pacientes que presentaron inadecuadas relaciones oclusales. De los pacientes que presentaron adecuadas relaciones oclusales la mayoría recibieron el tratamiento de desgastes proximales.

Heusdens, Dermaut y Verbeeck (17) realizaron un estudio experimental en el modelo de un paciente masculino que recibió tratamiento de ortodoncia sin extracciones al cual se le realizaron diferentes set-up y se encontraron que en los casos que presentaron una discrepancia de Bolton severa obtuvieron una relación molar de clase I, un overjet y un overbite aceptable es decir la discrepancia de Bolton severa afectó ligeramente a la oclusión.

Una limitación de este estudio puede ser el haberse realizado en modelos estáticos y sin el uso de radiografías; sin embargo, sus conclusiones son importantes debido a que en la actualidad no existen estudios acerca del tratamiento en la fase de acabado que deben recibir los pacientes para corregir la discrepancia de Bolton, y su asociación con las relaciones oclusales al final del tratamiento.

Se recomienda analizar en posteriores investigaciones la angulación inter-incisal a través de radiografías cefalométricas debido a que puede influenciar en el overbite, el overjet y las relaciones oclusales finales.

\section{Conclusiones}

- La distribución de la discrepancia de masa dentaria anterior fue mayor a la discrepancia total.

- La discrepancia de masa dentaria total inicial se ubicó con mayor frecuencia en la arcada superior y la discrepancia anterior inicial en la arcada inferior.

- El procedimiento clínico más utilizado para solucionar la discrepancia de Bolton fue el desgaste proximal y con ello, la discrepancia de masa dentaria final se sitúo entre los rangos reportados por Bolton.

- Finalizado el tratamiento, se obtuvieron relaciones oclusales aceptables, tuvieran ó no discrepancia de masa dentaria inicial.

\section{Referencias bibliográficas}

1. Tong H, Chen D, Xu L, Liu P. The effect of premolar extractions on tooth size discrepancies. Angle Orthod. 2004; 74(4):508-11.

2. Bolton WA. Disharmony in tooth size and its relation to the analysis and treatment of malocclusion. Angle Orthod. 1958; 28(3):11330.

3. Othman SA, Harradine NW. Tooth-size discrepancy and Bolton's ratios: a literature review. J Orthod. 2006; 33(1):4551.

4. Santoro M, Ayoub ME, Pardi VA, Cangialosi TJ. Mesiodistal crown dimensions and tooth size discrepancy of the permanent dentition of Dominican Americans. Angle Orthod. 2000; 70(4):303-7.

5. Stifter J. A Study Of Pont's, Howes', Rees', Neff's And Bolton's Analyses On Class I Adult Dentitions. 1958; 28(4): 215-55.

6. Bernabé E, Major PW, FloresMir C. Tooth-width ratio discrepancies in a sample of Peruvian adolescents. Am J Orthod Dentofacial Orthop. 2004; 125(3):361-5.

7. Santoro M, Ayoub ME, Pardi VA, Cangialosi TJ. Mesiodistal crown dimensions and tooth size discrepancy of the permanent dentition of Dominican Americans. Angle Orthod. 2000; 70(4):303-7.

8. Sheridan JJ, Hastings J. Air-rotor stripping and lower incisor extraction treatment. J Clin Orthod. 1992; 26(1):18-22.

9. Sheridan JJ. Air-rotor stripping. J Clin Orthod. 1985; 19(1):43-59.

10. Sheridan JJ. Air-rotor stripping update. J Clin Orthod. 1987; 21(11):781-8.

11. Joseph VP, Rossouw PE, Basson NJ. Orthodontic microabrasive reproximation. Am J Orthod Dentofacial Orthop. 1992; 102(4):351-9.

12.Fields HW Jr. Orthodonticrestorative treatment for relative mandibular anterior excess toothsize problems. Am J Orthod. 1981; 79(2):176-83.

13.Tuverson DL. Anterior interocclusal relations. Part I. Am J Orthod. 1980; 78(4):361-70.

14. Tuverson DL. Anterior interocclusal relations. Part II. Am J Orthod. 1980; 78(4):37193.

15.Cuoghi OA, Sella RC, Macedo 
FA, Mendonça MR. Desgaste interproximal e suas implicações clínicas. Rev Dent Press Ortodon Ortop Facial. 2007;12(3):32-46.

16. Uysal T, Sari Z, Basciftci FA, Memili B. Intermaxillary tooth size discrepancy and malocclusion: is there a relation? Angle Orthod. 2005; 75(2):20813.

17. Heusdens $M$, Dermaut L, Verbeeck R. The effect of tooth size discrepancy on occlusion: An experimental study. Am J Orthod Dentofacial Orthop. 2000; 117(2):184-91.

18. Rudolph DJ, Dominguez PD,
Ahn K, Thinh T. The use of tooth thickness in predicting intermaxillary tooth-size discrepancies. Angle Orthod. 1998; 68(2):133-8.

19.Al-Tamimi T, Hashim HA. Bolton tooth-size ratio revisited. World J Orthod. 2005; 6(3):28995.

20.Freeman JE, Maskeroni AJ, Lorton L. Frequency of Bolton tooth-size discrepancies among orthodontic patients. Am J Orthod Dentofacial Orthop. 1996; 110(1):24-7.

21.Araujo E, Souki M. Bolton anterior tooth size discrepancies among different malocclusion groups. Angle Orthod. 2003; 73(3):307-13.

22. Ramos AL, Suguino R, Terada HH, Furquim LZ, Filho OGS. Considerações sobre análise da discrepância dentária de Bolton e a finalização ortodôntica. Rev Dent Press Ortodon Ortop Facial. 1996 ; 1(2):86-106.

23.Li A. Orthodontic Treatment of 41 Patients with Tooth Size Discrepancy. Life Sci J. 2006 ; 3(4):65-7.

24. Moreno M. Interproximal Enamel Reduction. World J Orthod. 2002; 3(3):223-32. 